\title{
Preliminary Results from Clinical Validation Study of a Method for Non-Invasive Assessment of Atrioventricular Node Refractoriness during Atrial Fibrillation
}

\author{
Frida Sandberg ${ }^{1}$, Valentina D A Corino ${ }^{2}$, Leif Sörnmo ${ }^{1}$, Pyotr Platonov ${ }^{3}$, Fredrik Holmqvist ${ }^{3}$ \\ ${ }^{1}$ Department of Biomedical Engineering and Center for Integrative Electrocardiology (CIEL), Lund \\ University, Lund, Sweden, \\ ${ }^{2}$ Dipartimento di Elettronica, Informazione e Bioingegneria, Politecnico di Milano, Milan, Italy \\ ${ }^{3}$ Department of Cardiology and Center for Integrative Electrocardiology (CIEL), Lund University, \\ Lund, Sweden
}

\begin{abstract}
The purpose of this study is to evaluate our previously proposed model-based, non-invasive approach to assess atrioventricular $(A V)$ node refractory periods during atrial fibrillation $(A F)$ by comparing its estimates to the invasively assessed $A V$ node refractory periods. Patients referred to the hospital for pulmonary vein isolation (PVI), being in AF upon arrival, are included in the study. Using our approach, we estimate the AV node refractory periods from the ECG before and during the PVI procedure. The refractory periods is estimated using both single and dual pathway models; Bayes Information Criterion is employed to select the most appropriate model. Following conversion to sinus rhythm, a SIS2 atrial pacing protocol is applied to invasively assess AV node refractory periods. Preliminary results suggest that the method for non-invasive assessment of AV node refractoriness is accurate, however, more data is needed to better establish performance.
\end{abstract}

\section{Introduction}

During atrial fibrillation (AF), the atrioventricular (AV) node is continuously bombarded with atrial electrical impulses. The AV node is the natural barrier which restricts conduction of atrial impulses into the His-Purkinje system and is therefore very important during AF since it to some extent protects the ventricles of the heart from the extremely fast atrial rate. Electrophysiologic factors such as refractoriness of the AV node and concealed conduction influence the $\mathrm{AV}$ nodal blocking of impulses. Even though these properties play a prominent role in ventricular rate control, they are not routinely evaluated in clinical practice. The existence of dual AV nodal pathways, i.e., a fast pathway (FP) with a longer refractory period and a slow pathway (SP) with shorter refractory period [1] is a further complicating factor. During normal sinus rhythm atrial impulses are generally conducted though the FP, but during AF conduction may occur through the SP due to its shorter refractory period.

We have previously proposed a model-based approach for joint analysis of atrial and ventricular activity in the ECG during AF [2-4]. The method is based on a functional model of the AV node where the model parameters reflects SP and FP refractoriness. The method has been used to analyze 12-lead resting ECG data from 14 patients in a phase II, open-label, sequential-group, doseescalation trial of tecadenoson administered intra venously alone and in combination with esmolol [5] as well as longterm ECG data from 60 patients in a randomized, crossover study comparing the efficacy of four different rate control drugs [6]. The results showed that changes in AV node refractoriness, as reflected in the model parameter estimates, were in agreement with previous clinical findings. This suggests that the model parameters estimated from ECG during AF may be used as a surrogate for the AV node refractory period invasively assessed through a pacing protocol [7].

The purpose of the present study is to evaluate our model-based, non-invasive approach to assess AV node refractory periods during AF by comparing its estimates to the invasively assessed AV node refractory periods. Patients referred to the hospital for pulmonary vein isolation (PVI), being in AF upon arrival, are included in the study. Using our approach, we estimate the SP and FP refractory periods from the ECG, which is recorded for approximately 2 hours before and during the PVI procedure. Following conversion to sinus rhythm, an S1S2 atrial pacing protocol is applied to invasively assess the AV node refractory periods. Here we present preliminary results from the first two patients included in the study. 


\section{Methods}

\subsection{Model}

In our model [2-4], the AV node is treated as a lumped structure which accounts for concealed conduction, relative refractoriness, and dual AV nodal pathways. Atrial impulses are assumed to arrive to the AV node according to a Poisson process with mean arrival rate $\lambda$. Each arriving impulse immediately results in ventricular activation unless blocked by a refractory AV node.

The probability of an atrial impulse passing through the AV node depends on the time elapsed since the previous ventricular activation. The refractory period is defined by a deterministic part $\tau$ and a stochastic part, the latter part modeling prolongation due to concealed conduction and/or relative refractoriness and assumed to be uniformly distributed over the interval $\left[0, \tau_{p}\right]$. Hence, all atrial impulses arriving to the AV node before the end of the refractory period $\tau$ are blocked. Then follows an interval $\left[\tau, \tau+\tau_{p}\right]$ with linearly increasing likelihood of penetration into the AV node. Finally, no impulses are blocked arriving after the end of the maximally prolonged refractory period $\tau+\tau_{p}$.

The refractory period length is assumed to be different depending on the penetrating pathway; the SP refractory period is defined by the parameters $\tau_{s}$ and $\tau_{p, s}$, whereas the FP refractory period is defined by $\tau_{f}$ and $\tau_{p, f}$. The probability of an atrial impulse being conducted through the SP is equal to $\alpha$, and accordingly the probability of conduction through the SP is $(1-\alpha)$. The SP refractory period is shorter than that of the FP, $\tau_{s} \leq \tau_{f}$.

For this model, the time intervals $x_{i}$ between consecutive ventricular activations, i.e., corresponding to the RR intervals, are independent. It can be shown that the joint probability density function (PDF) is given by [2]

$$
\begin{aligned}
p_{x}\left(x_{1}, x_{2}, \ldots, x_{M}\right) & = \\
& \prod_{m=1}^{M}\left(\alpha p_{x, s}\left(x_{m}\right)+(1-\alpha) p_{x, f}\left(x_{m}\right)\right),
\end{aligned}
$$

where $M$ is the total number of intervals, and $p_{x, i}\left(x_{m}\right), i \in$ $\{f, s\}$, is given by

$p_{x, i}(x)=\left\{\begin{array}{l}0, \quad x<\tau_{i} \\ \frac{\lambda\left(x-\tau_{i}\right)}{\tau_{p, i}} \exp \left\{\frac{-\lambda\left(x-\tau_{i}\right)^{2}}{2 \tau_{p, i}}\right\}, \quad \tau_{i} \leq x<\tau_{i}+\tau_{p, i} \\ \lambda \exp \left\{\frac{-\lambda \tau_{p, i}}{2}-\lambda\left(x-\tau_{i}-\tau_{p, i}\right)\right\}, \quad x \geq \tau_{i}+\tau_{p, i} .\end{array}\right.$
To account for the interdependence between successive RR intervals, the deterministic part of the refractory period is assumed to depend on the preceding RR interval, so that a longer RR interval is followed by a longer refractory period, and vice versa.

\subsection{Model paremeter estimation}

The model parameters are estimated from $15 \mathrm{~min}$, $50 \%$ overlaping segments of ECG. The arrival rate of atrial impulses $\lambda$ is estimated from the atrial activity of the ECG, obtained using spatiotemporal QRSTcancellation [8]. First, an AF frequency trend with 10-s resolution is estimated for the whole recording using an HMM-based approach [9]. Then, $\lambda$ is taken as the median $\mathrm{AF}$ frequency within the segment of interest, after correction to account for atrial refractoriness [3].

All other model parameters, namely $\alpha, \tau_{s}, \tau_{f}, \tau_{p, s}, \tau_{p, f}$, are estimated from the RR series by maximizing joint PDF in eq. (1) with respect to $\boldsymbol{\theta}=\left[\begin{array}{lllll}\alpha & \tau_{s} & \tau_{f} & \tau_{p, s} & \tau_{p, f}\end{array}\right]^{T}$. Prior to the maximum likelihood (ML) estimation, RR intervals adjacent to beats with abnormal morphology, such as ectopic beats and artifacts, are removed from the RR series. Since the property of statistical independence is not fully valid for observed RR intervals, approximate decorrelation is performed prior to ML-estimation to reduce the interdependence between subsequent RR intervals. The parameters of a single pathway model, $\left[\begin{array}{ll}\tau & \tau_{p}\end{array}\right]^{T}$ are also estimated. The Bayes information criterion is used to determine the most appropriate model [3]. Since no closedform solution could be found for $\hat{\boldsymbol{\theta}}$, combined with the fact that the gradient is discontinuous, the multi-swarm particle swarm optimization (MPSO) is used to optimize the loglikelihood function [10].

\section{Study Protocol}

The study was approved by the local research ethics committee, and informed consent was obtained from all subjects. The study population consists of patients referred to Skåne University Hospital in Lund, Sweden, for PVI. Patients that are in AF upon arrival to the hospital are included in the study. We plan to enroll 25 patients in the study; here we present preliminary results from the first 2 patients. The ECG was recorded throughout the PVI procedure, approximately $1-2$ hours. Following conversion to sinus rhythm, a standard S1S2 atrial pacing protocol was applied to invasively assess AV node refractory periods. The basic pacing cycle length was set to $500 \mathrm{~ms}$ and the test cycle length was successively decremented by $10 \mathrm{~ms}$ until conduction through the AV node was no longer observed. The effective refractory period is defined as the longest non-conducing S1S2. The functional refractory 
period (FRP) is defined as the shortest time between conducted impulses; in this study we approximate it as the shortest S1S2 that results in a ventricular activation. If the conduction curve contains a "jump", i.e. an abrupt increase $\geq 50 \mathrm{~ms}$ in conduction delay in response to a S1S2 shortening of $10 \mathrm{~ms}$, the patient has dual AV nodal pathways physiology according to the clinical definition.

\section{Preliminary results}

\subsection{Clinical parameters}

None of the two patients currently enrolled in the study had dual pathway physiology according to the clinical definition. The invasively assessed FRP was $320 \mathrm{~ms}$ and 370 ms for patient \#1 and \#2, respectively.

The average heart rate prior to sinus rhythm conversion was $119 \mathrm{bpm}$ and $92 \mathrm{bpm}$ for patient \#1 and patient \#2, respectively. The corresponding AF frequency (AF rate) was $6.6 \mathrm{~Hz}(396 \mathrm{fpm})$ and $5.0 \mathrm{~Hz}(300 \mathrm{fpm})$, respectively. Trends of heart rate and AF rate estimated from the ECG during the PVI procedure prior to sinus rhythm conversion is displayed in Fig. 1. The heart rate increased during the procedure for both patients; from $118 \mathrm{bpm}$ in the first 15min segment to $135 \mathrm{bpm}$ in the last 15 -min prior to $\mathrm{AF}$ termination for patient $\# 1$ and from $77 \mathrm{bpm}$ in the first 15 -min segment to $100 \mathrm{bpm}$ in the last 15 -min prior to $\mathrm{AF}$ termination for patient \#2. The AF frequency decreased throughout the procedure for patient $\# 1$, from initial 7.1 $\mathrm{Hz}$ to $5.9 \mathrm{~Hz}$. For patient \#2 the AF frequency was between 5.0 and $5.2 \mathrm{~Hz}$ until the final 15 -min prior to sinus rhythm conversion when it decreased to $4.7 \mathrm{~Hz}$.

\section{2. $\quad$ Estimated Model Parameters}

Examples of decorrelated RR interval histograms from 15-min segments of ECG and corresponding estimated model PDFs are displayed in Fig. 2. In Fig. 2(a), a dual pathway model was selected and the estimated model parameters were $\tau_{s}=306 \mathrm{~ms}, \tau_{f}=352 \mathrm{~ms}, \tau_{p, s}=217 \mathrm{~ms}$, $\tau_{p, f}=64 \mathrm{~ms}$, and $\alpha=0.37$. In Fig 2(b) a single pathway model was selected and the estimated model parameters were $\tau=475 \mathrm{~ms}, \tau_{p}=330 \mathrm{~ms}$.

A single pathway model was selected in $56 \%$ of the analyzed segments for patient \#1 and in all analyzed segments for patient \#2. In the remaining segments, a dual pathway model was selected. For patient \#1, the estimates of $\tau$ in segments where the single pathway model was $323 \pm 12$ ms (mean \pm std), whereas the estimates of $\tau_{s}$ and $\tau_{f}$ when the dual pathway model was selected was $307 \pm 6 \mathrm{~ms}$ and $362 \pm 12 \mathrm{~ms}$, respectively. The difference between $\tau_{s}$ and $\tau_{f}$ when the dual pathway model was selected was small, $56 \pm 11 \mathrm{~ms}$. For patient $\# 2$, the single pathway model was

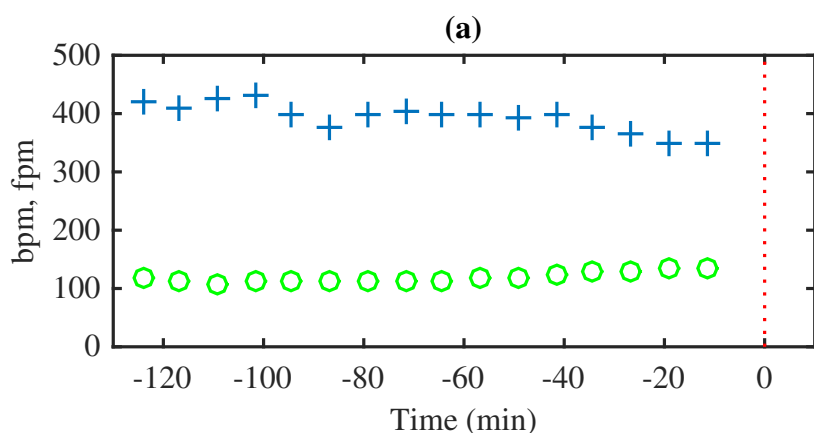

(b)

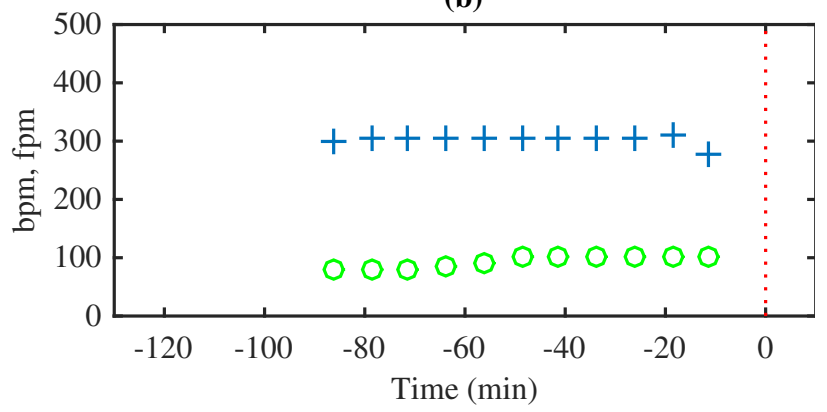

Figure 1. ('o') Heart rate and ('+') AF rate during PVI procedure, prior to (dotted line) sinus rhythm conversion.

selected in all segments and the estimate of $\tau$ was $421 \pm 44$ ms.

\subsection{Comparison}

Trends of the estimated model parameters during the PVI procedure and invasively assessed FRP are displayed in Fig 3. For patient $\# 1, \tau$ (and $\tau_{s}$ when the dual pathway model is selected) was similar throughout the PVI procedure, ranging from 299 to $341 \mathrm{~ms}$. For patient \#2, $\tau$ decreased during the PVI procedure, from $494 \mathrm{~ms}$ in the beginning to $396 \mathrm{~ms}$ at the end. Note that FPR is assessed after conversion to sinus rhythm, and that the time between the last $\tau$ estimate and the invasively assessed FPR is approximately 20 minutes.

For patient \#1, the invasively assessed FRP was $320 \mathrm{~ms}$ whereas $\tau$ estimated from the last 15-min prior to sinus rhythm conversion was $299 \mathrm{~ms}$. The difference between this value of $\tau$ and the invasively assessed FRP, $21 \mathrm{~ms}$, was in the same order of magnitude as the maximal difference between $\tau$ estimated from consecutive 15-min segments, $22 \mathrm{~ms}$. For patient \#2, the invasively assessed FRP was $370 \mathrm{~ms}$ whereas $\tau$ estimated from the last 15-min prior to sinus rhythm conversion was $396 \mathrm{~ms}$. The difference between this value of $\tau$ and the invasively assessed FRP, $26 \mathrm{~ms}$, was smaller than the maximal difference between $\tau$ estimated from consecutive 15 -min segments, $87 \mathrm{~ms}$. 
(a)

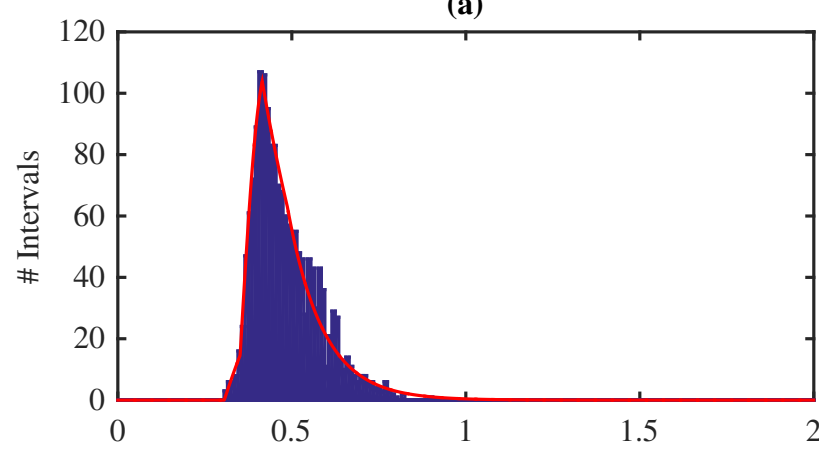

(b)

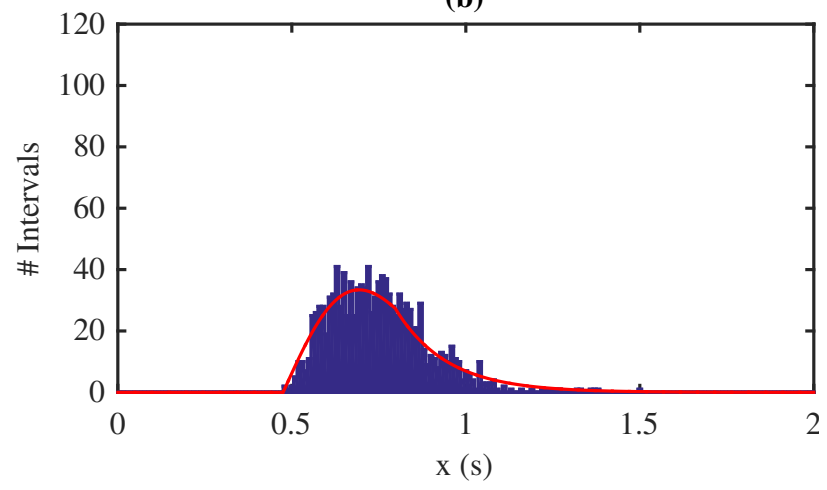

Figure 2. (blue) Histograms of decorrelated RR series and (red) estimated model PDF from 15-min of ECG from (a) patient \#1 and (b) patient \#2.

\section{Conclusions}

Preliminary results suggest that the method for noninvasive assessment of AV node refractoriness produces accurate results. More data is needed for conclusive results.

\section{References}

[1] Kurian T, Ambrosi C, Hucker W, Fedorov VV, Efimov IR. Anatomy and electrophysiology of the human av node. Pacing Clin Electrophysiol 2010;33:754-762.

[2] Corino VDA, Sandberg F, Mainardi L, Sörnmo L. An atrioventricular node model for analysis of the ventricular response during atrial fibrillation. IEEE Trans Biomed Eng 2011;58:3386-3395.

[3] Corino VDA, Sandberg F, Lombardi F, Mainardi L, Sörnmo L. Atrioventricular nodal function during atrial fibrillation: Model building and robust estimation. Biomed Signal Process Control 2013;8(6):1017-1025.

[4] Henriksson M, Corino V, Sörnmo L, Sandberg F. A Statistical Atrioventricular Node Model Accounting for Pathway Switching During Atrial Fibrillation. IEEE Trans Biomed Eng 2016;63:1842-1849.

[5] Corino VDA, F., Mainardi LT, Platonov PG, Sörnmo L.
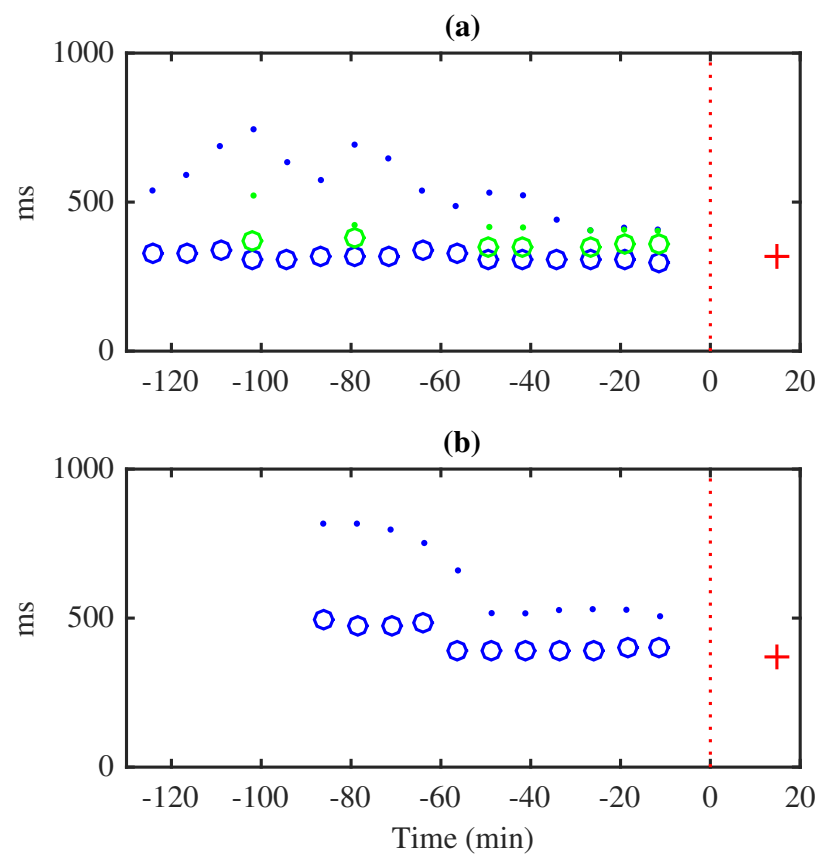

Figure 3. Model parameter estimates and ('+') invasively assessed FRP from (a) patient \#1 and (b) patient \#2. ('o') $\tau$ and ('.') $\tau+\tau_{p}$ during PVI procedure prior to (dotted line) sinus rhythm conversion. In segments where a dual pathway model is selected, blue denotes $\tau_{s}$ and $\tau_{s}+\tau_{p, s}$ whereas green denotes $\tau_{f}$ and $\tau_{f}+\tau_{p, f}$.

Noninvasive assessment of atrioventricular nodal function: Effect of rate-control drugs during atrial fibrillation. Ann Noninvasive Electrocardiol 2015;20(6):534-41.

[6] Sandberg F, Corino V, Mainardi LT, Ulimoen S, Enger S, Tveit A, Platonov P, Sörnmo L. Non-invasive assessment of the effect of beta blockers and calcium channel blockers on the AV node during permanent atrial fibrillation. J Electrocardiol 2015;48(5):861-6.

[7] Billette J, Tadros R. Integrated rate-dependent and dual pathway $\mathrm{AV}$ nodal functions: principles and assessment framework. Am J Physiol Heart Circ Physiol 2014; 306:H173-83.

[8] Stridh M, Sörnmo L. Spatiotemporal QRST cancellation techniques for analysis of atrial fibrillation. IEEE Trans Biomed Eng 2001;48(1):105-111.

[9] Sandberg F, Stridh M, Sörnmo L. Frequency tracking of atrial fibrillation using hidden Markov models. IEEE Trans Biomed Eng 2008;55:502-511.

[10] Niu B, Zhu Y, He X, Wu H. Mcpso: A multi-swarm cooperative particle swarm optimizer. Appl Math Comput 2007; 2:1050-1062.

Address for correspondence:

Frida Sandberg

frida.sandberg@bme.lth.se 